\title{
L'éducation relative au changement climatique : une lecture à la lumière du Pacte de Glasgow
}

Adolfo Agundez-Rodriguez et Lucie Sauvé

\section{(2) OpenEdition}

Édition électronique

URL : https://journals.openedition.org/ere/8421

ISSN : 2561-2271

\section{Éditeur}

Centr'ERE

\section{Référence électronique}

Adolfo Agundez-Rodriguez et Lucie Sauvé, «L'éducation relative au changement climatique : une lecture à la lumière du Pacte de Glasgow », Éducation relative à l'environnement [En ligne], Volume 17-1 | 2022, mis en ligne le 27 janvier 2022, consulté le 03 février 2022. URL : http:// journals.openedition.org/ere/8421

Ce document a été généré automatiquement le 3 février 2022

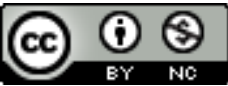

La revue Éducation relative à l'environnement est mise à disposition selon les termes de la Licence Creative Commons Attribution - Pas d'Utilisation Commerciale 4.0 International. 


\title{
L'éducation relative au changement climatique : une lecture à la lumière du Pacte de Glasgow
}

\author{
Adolfo Agundez-Rodriguez et Lucie Sauvé
}

1 Depuis quarante ans, le changement climatique ${ }^{1}$ (CC) s'est accéléré de façon alarmante en raison des émissions de gaz à effet de serre d'origine anthropique dans le contexte des sociétés de surconsommation (Groupe d'experts intergouvernemental sur l'évolution du climat - GIEC, 2021). Le CC appauvrit les écosystèmes des cinq continents et ceux des océans, mettant en danger la vie sur Terre. Ce phénomène constitue non seulement le plus grand défi écologique que l'humanité doit affronter actuellement, mais aussi le plus grand défi social (GIEC, 2021 ; Incropera, 2016). Le CC affecte notre santé, réduit l'accessibilité à l'eau, menace la biodiversité, nuit à la sécurité alimentaire, exacerbe les inégalités, entraîne l'intensification des conflits et des déplacements massifs de populations (Welzer, 2012). La réduction des sources de gaz à effet de serre devient donc une exigence mondiale pour atténuer le CC (GIEC, 2021). Or l'éducation relative au changement climatique (ERCC) peut jouer un rôle de premier plan pour donner accès aux connaissances, développer un jugement critique et un pouvoir-agir, de façon à inspirer et soutenir des actions visant l'atténuation du CC (Anderson et Brows, 2011 ; Didham, Doyle, Klein et Thoresen, 2015). La formation des personnes élu.e.s, du grand public et des jeunes en ce sens constitue une priorité (CCNUCC Convention-cadre des Nations unies sur les changements climatiques, 2015; Riechmann, 2015).

2 Depuis l'adoption de l'Accord de Paris (CCNUCC, 2015), l'éducation est en effet reconnue à l'échelle internationale comme un outil essentiel d'information, de sensibilisation et de participation du public à la lutte contre le CC (Article 12). L'ERCC devient ainsi un domaine fondamental et transdisciplinaire de recherche et de formation des citoyens et citoyennes du XXI ${ }^{e}$ siècle, faisant appel à toutes les disciplines de la connaissance. En tant que domaine transversal, l'ERCC devrait également être soutenue par les diverses politiques publiques d'ordre social, environnemental, énergétique et éducatif, dans 
tous les pays du monde. L'ERCC a en effet pour visée de favoriser un meilleur vivre ensemble sur Terre dans le contexte actuel qui fait appel à une profonde transformation écosociale.

3 Dans la foulée de l'Accord de Paris (CCNUCC, 2015), le Pacte de Glasgow (CCNUCC, 2021) a réitéré l'importance de l'ERCC pour limiter le réchauffement à 1,5 degré Celsius. Parmi les aspects fondamentaux abordés lors de la Conférence de Glasgow qui devraient, entre autres, être abordés par l'ERCC se trouvent 1) les causes et les conséquences du changement climatique, 2) l'augmentation des pertes (humaines, économiques, environnementales) associées au CC et plus spécifiquement, à la consommation de combustibles fossiles, 3) le drame de l'injustice écosociale et ses conséquences, 4) le fonctionnement et la normativisation des marchés du carbone.

En effet, l'ERCC doit premièrement devenir un espace éducatif où les citoyennes et citoyens sont informé.e.s et sensibilisé.e.s aux causes et aux conséquences du CC, en mettant l'accent sur l'augmentation anormale des événements météorologiques extrêmes et les pertes de toutes sortes qu'ils entraînent pour la planète, pour le monde vivant, dont l'humanité tout entière. En ce sens, l'ERCC doit faire écho au fait que le bénéfice économique associé aux actions humaines qui causent le changement climatique est actuellement inférieur aux pertes économiques, environnementales et humaines que celles-ci produisent. Parmi des centaines d'exemples, mentionnons les deux suivants signalés par Castillo (2016) : le renforcement des digues de la NouvelleOrléans après l'ouragan Katrina en 2005 ayant causé la mort de1 800 personnes, a coûté 4,5 milliards de dollars; le gouvernement des Pays-Bas prévoit dépenser 1000 millions d'euros par année pour retenir la montée du niveau de la mer qui menace d'inonder ses terres.

5 Deuxièmement, l'ERCC doit contribuer à la reconnaissance de la gravité des conséquences de la poursuite de la consommation d'énergies fossiles associées aux activités humaines primaires (agriculture, élevage), secondaires (activités industrielles basées sur les matières premières) et tertiaires (services tels que le tourisme). Par ailleurs, l'ERCC doit dénoncer le fait que cette gravité augmente lorsqu'on passe d'une logique de consommation à une logique d'hyperconsommation typique des sociétés riches d'un point de vue économique. En ce sens, certaines activités, telles que l'exploitation de monocultures, l'élevage intensif, la production d'objets superflus et le tourisme de masse, doivent être présentées comme contrevenant à la stabilité climatique et nuisibles à la vie sur Terre. Afin de limiter le réchauffement à 1,5 degré Celsius, le Pacte de Glasgow (CCNUCC, 2021) mentionne l'importance de freiner tous les nouveaux projets d'exploitation d'énergies fossiles au moins jusqu'en 2050. En ce sens, l'ERCC, en dénonçant énergiquement l'utilisation des hydrocarbures, s'inscrit dans les orientations de la CCNUCC.

Troisièmement, l'ERCC doit être un espace de dénonciation des injustices socioclimatiques. Le Pacte de Glasgow (CCNUCC, 2021) suppose un engagement des États du monde à financer - à la hauteur de100 milliards de dollars américains par année -, la lutte contre le CC dans les pays les plus pauvres. Cette mesure est fondée sur la reconnaissance par les États que, lorsqu'il s'agit du CC, le partage des responsabilités et des coûts doit être inégal pour être équitable. En ce sens, les pays pauvres, qui ont le moins contribué à la crise climatique et qui sont souvent les plus vulnérables à ses effets, ne devraient pas, par ailleurs, en payer le prix. De plus, c'est une question de justice socioclimatique que les dommages, souvent irréversibles (surtout lorsqu'ils 
fauchent des vies humaines ou favorisent l'extinction d'espèces) soient assumés par les pays qui ont émis le plus de $\mathrm{CO} 2$ dans l'atmosphère.

Quatrièmement, l'ERCC devrait contribuer à développer une réflexion critique autour de la création, la gestion et le fonctionnement des marchés du carbone. D'une part, il s'agit d'inviter les citoyennes et citoyens à examiner la manière dont ces marchés d'échange, d'achat et de vente du droit d'émettre des gaz à effet de serre sont normés. D'autre part, il s'agit de mettre en lumière le fait que de nombreuses entreprises et multinationales bénéficient financièrement du CC. Comme l'observe Castillo (2016), parmi les types d'entreprises qui accumulent le plus de profits grâce au CC figurent les entreprises d'ingénierie et de construction (responsables de la restauration des territoires dévastés par des événements météorologiques extrêmes), les sociétés pharmaceutiques (pour lutter contre l'augmentation de la fréquence des maladies causées par le CC, comme les maladies pulmonaires en général dont l'asthme et les problèmes de santé mentale associés à l'écoanxiété), l'industrie agro-alimentaires (qui produit des engrais pour enrichir les terres agricoles appauvries) et celle de l'armement (responsable de la production et de la vente d'armes dans le cadre de conflits territoriaux et pour défendre les frontières «menacées " par la migration forcée). Ainsi, les marchés associés au CC contribuent à accentuer l'injustice écosociale. Les pays les plus pauvres, qui ont le moins contribué au CC, sont souvent ceux qui souffrent davantage les conséquences. L'absence de ressources, la dévastation et la perte de fertilité de leurs territoires, la contamination de leurs sols agricoles et l'augmentation des maladies génèrent ou aggravent des conflits territoriaux dus au manque d'accès aux ressources vitales, comme la nourriture et l'eau, les rendent dépendants des pays plus compétitifs sur la scène économique dominante. Face à cette situation, les pays les plus riches, premiers producteurs d'engrais, de médicaments et d'armes, s'enrichissent grâce au marché du CC qu'ils génèrent. Dans cette situation, l'injustice socioclimatique s'accroît et le problème du CC est loin d'être résolu. Sur la base de l'information et de la sensibilisation sur les principaux aspects du CC, l'ERCC vise donc également à stimuler l'émergence d'alternatives et de solutions à une telle problématique.

8 Ainsi, à travers le développement de la pensée critique, l'ERCC met en lumière les aspects les plus sombres du CC et enrichit le débat de manière transdisciplinaire en tenant compte des différents points de vue. Mais plus encore, l'ERCC invite à repérer, à partager, à promouvoir et enrichir les solutions déjà en cours dans de nombreuses régions $\mathrm{du}$ monde, ainsi qu'à générer de nouvelles pratiques pour modifier radicalement la situation actuelle et inspirer de nouveaux modes de vie. L'ERCC peut stimuler l'émergence d'initiatives dans les domaines du transport, de l'agroalimentaire, de l'habitation, de l'aménagement du territoire et de la consommation en général, visant la sobriété énergétique, privilégiant des technologies alternatives aux hydrocarbures, se préoccupant du cycle de vie des matériaux et produits, et respectant la dynamique des écosystèmes naturels. Ici, le bien commun, celui de toute l'humanité et du monde vivant dans son ensemble, doit primer sur les intérêts des individus, des gouvernements et des sociétés transnationales.

9 Une telle vision de l'ERCC peut se déployer dans les divers contextes d'apprentissage : en milieu formel (écoles, collèges et universités), non formel (organismes environnementaux, musées, parcs, etc.) et informel (actions éducatives non planifiées qui se déroulent spontanément dans la société, comme dans le cadre de mouvements 
sociaux). Le Pacte de Glasgow (CCNUCC, 2021) propose en effet six créneaux pour développer le pouvoir d'action face à la crise climatique : 1) l'accès à l'information, y compris la production de matériel pédagogique facilement accessible en libre accès, ainsi que leur publication dans différentes langues, y compris les langues des régions les plus vulnérables aux CC ; 2) l'éducation (formelle et non formelle) ; 3) la formation universitaire et la formation d'adultes, gouverneurs, dirigeants communautaires, directeurs d'université et travailleurs de tous les domaines et disciplines (professeurs, journalistes, scientifiques, etc.) ; 4) la sensibilisation de citoyennes et citoyens par tous les moyens de communication possibles ; 5) la participation des citoyennes et citoyens de tous âges à la vie publique, aux négociations et à la prise de décisions concernant le climat (éducation informelle), notamment par le biais de consultations fréquentes sur les mesures prises en matière de changement climatique aux niveaux national et international; 6) la coopération internationale entre les organisations, les gouvernements, les institutions et les mouvements sociaux dans la lutte contre le changement climatique.

Selon le Pacte de Glasgow (CCNUCC, 2021), ce n'est qu'à travers ces six stratégies que les buts et objectifs fixés par l'Accord de Paris (CCNUCC, 2015) peuvent être atteints. Chaque État est doté d'autonomie pour intégrer de manière flexible les lignes directrices du Pacte dans son système éducatif, en collaboration avec d'autres États et selon une approche holistique, intergénérationnelle et interdisciplinaire, et qui intègre les questions d'équité, dont celles liées au genre. Plus encore, mentionnant explicitement l'importance de s'inspirer des connaissances autochtones pour l'ERCC, le Pacte de Glasgow (CCNUCC, 2021) privilégie la transdisciplinarité.

11 Comme source d'inspiration pour le développement de l'ERCC, il convient de souligner ici la pertinence des mouvements de jeunes qui se sont déployés au cours des dernières années. En effet, la prise de conscience du changement climatique chez les jeunes, comme au sein de la population en général, s'est considérablement accrue grâce entre autres à l'impact international du mouvement étudiant Fridays for the futur (Vendredis pour l'avenir), initié en Suède en août 2018 par Greta Thunberg.

Depuis lors et jusqu'en mars 2020 où la pandémie a changé nos vies, des manifestations étudiantes ont eu lieu chaque vendredi dans des dizaines de villes du monde entier. Un temps fort de ce mouvement a eu lieu le 15 mars 2019, avec plus de 2000 manifestations d'élèves et d'étudiants dans 125 pays. C'est au Québec que le rassemblement a été le plus important, avec près de 500000 manifestants (Léveillé, 2019). Les jeunes se sont principalement adressés aux gouvernements, exigeant que des lois soient adoptées et que des mesures soient prises pour inverser la situation actuelle $\mathrm{du}$ CC. Leur discours était éminemment politique et s'adressait surtout aux dirigeants des pays riches. Outre la modification des modes de production, de transport et de consommation visant à réduire les émissions de gaz à effet de serre, les jeunes ont demandé à leurs gouvernements de faire preuve de transparence dans un contexte de libre marché qui favorise les inégalités entre une minorité de riches et une trop grande partie de la population mondiale vivant dans la misère. En ce sens, le discours des jeunes dépassait la demande de mesures visant à arrêter la hausse des températures : il traduisait une critique des choix politiques et économiques dominants et de leurs effets sur les populations, en particulier les plus défavorisées. Ce mouvement a stimulé la naissance d'une nouvelle culture sociétale basée sur une conscience écosociale, en lien 
avec d'autres mouvements de justice sociale tels que le féminisme ou l'anticolonialisme.

13 En ce sens, les jeunes ont revendiqué l'intégration d'une éducation relative à l'environnement (ERE) dans les curriculums. Au Québec, des mobilisations, telles que La Planète s'invite à l'Université, Pour le futur (au secondaire), le Devoir environnemental collectif (DEC) au collégial, tout comme le mouvement des Profs pour la planète, sont nés dans le même sens et ont réclamé une plus grande institutionnalisation de l'ERE. Leurs demandes rejoignent étroitement celles des différentes organisations et personnes qui se sont regroupées au sein de la Coalition Éducation, Environnement et Écocitoyenneté ${ }^{2}$ pour présenter au gouvernement une proposition de Stratégie québécoise d'éducation en matière d'environnement et d'écocitoyenneté ${ }^{3}$. Celle-ci vise à « offrir des leviers adéquats pour intégrer une éducation relative à l'environnement et à l'écocitoyenneté dans le système éducatif québécois comme dans l'ensemble de notre société ", de façon à remédier à la crise climatique actuelle, entre autres.

14 En somme, le mouvement étudiant s'est déployé tant dans le cadre de l'éducation formelle (il est né et s'est développé dans les contextes scolaires) que dans celui de l'éducation non formelle (en collaboration avec des organisations non gouvernementales, par exemple) et informelle (en prenant la forme d'un mouvement social à travers les marches par exemple). Il investit ainsi les divers créneaux d'ERCC identifiés par le Pacte de Glasgow. Par ailleurs, ce mouvement des jeunes revendique la pensée critique comme élément indispensable pour aborder en particulier les causes et les conséquences du CC, l'injustice socioclimatique et l'enjeu de la normativité des marchés de carbone émergents. Il invite à l'engagement dans l'action. C'est pourquoi le mouvement des jeunes nous apparaît comme une pratique particulièrement inspirante d'ERCC pouvant nous servir de modèle.

15 Les écoles devraient être ainsi les premières à valoriser l'engagement climatique des jeunes. Une telle valorisation devrait se traduire par un soutien aux actions qu'ils proposent de plusieurs manières : 1 ) inclure dans l'horaire scolaire des moments leur permettant de discuter d'enjeux sociaux et environnementaux, de s'organiser et de se mobiliser pour le climat;2) faciliter l'utilisation des infrastructures scolaires à cet effet ; 3) sensibiliser les parents des élèves à l'importance de soutenir leurs enfants dans leurs actions en leur permettant, par exemple, de participer à des manifestations pour le climat pendant les heures de classe; 4) libérer le personnel enseignant et administratif des écoles de certaines tâches, afin de pouvoir accompagner les jeunes dans leurs revendications ; 5) construire des ponts avec les médias pour donner de la visibilité aux actions des jeunes pour le climat. Certes, d'autres formes de collaboration peuvent être également envisagées. Dans tous les cas, l'école doit montrer sa pleine confiance envers les jeunes d'aujourd'hui, en reconnaissant par son soutien, la valeur de leur action climatique, à la fois pour l'atténuation du CC et pour leur apport à la prise de conscience collective et mondiale de l'importance de cet enjeu. Aucun autre mouvement pour le climat n'a eu autant de visibilité que celui de jeunes, qui a mis en évidence l'inefficacité des États et des organisations internationales dans la lutte pour le climat. Cet inestimable apport de la jeunesse devrait être davantage célébré par les écoles, les ministères de l'Éducation, les gouvernements et la société civile du monde entier. En lien avec la valorisation du rôle des jeunes, l'ERCC doit être reconnue par les milieux éducatifs de tous ordres d'enseignement (au préscolaire, primaire et secondaire, en formation professionnelle et des adultes, à l'université) ainsi que par 
l'ensemble de la société, comme une éducation fondamentale du XXIe siècle qui répond au plus grand défi de l'humanité.

Comme acteurs du champ d'étude et de pratique de l'ERCC, nous nous questionnons sur les pas à franchir pour instaurer une telle action éducative qui aille au-delà de la transmission de connaissances et de l'adoption d'écogestes individuels, pour stimuler vers l'engagement collectif. À l'appui des mouvements écosociaux, tels que celui du Vendredi pour l'avenir, il faut reconnaitre toute la pertinence de comprendre le changement climatique comme phénomène politique et social. L'ERCC doit favoriser l'engagement citoyen en vue de l'émergence d'une nouvelle culture sociétale basée sur la justice socioclimatique globale.

Toutefois, cette ERCC est confrontée à des obstacles de type psychologique, comportemental et cognitif (Meira Cartea, 2008 ; Pruneau, Khattabi et Demers, 2008) dont, entre autres, le déni de la situation et de ses conséquences, la difficulté de changer des habitudes de surconsommation ou encore celle de comprendre la complexité des réalités climatiques. C'est pourquoi l'ERCC vise le développement de quatre champs de compétences intégrées entre elles: des compétences d'ordre scientifique, critique, éthique et politique (Sauvé, 2014; Agundez-Rodriguez, en rédaction). Alors que le développement de compétences d'ordre scientifique contribue à la compréhension des concepts et des théories centrales pour mieux appréhender le $\mathrm{CC}$, les compétences d'ordre critique, éthique et politique, quant à elles, contribuent au développement d'une écocitoyenneté compétente et engagée pour l'atténuation du CC et l'adaptation à une telle réalité globale.

Une telle approche de l'ERCC a inspiré la réalisation du colloque international L'éducation relative au changement climatique: principes, approches et pratiques en milieux formel et non formel, organisé par le Centr'ERE - Centre de recherche en éducation et formation relatives à l'environnement et à l'écocitoyenneté ${ }^{4}$ - en octobre $2019^{5}$. Dans le cadre de ce numéro thématique, nous avons récupéré l'esprit du colloque et lancé un appel à contribution à l'ensemble de la communauté internationale en éducation relative à l'environnement.

19 Les douze textes de ce numéro thématique traitent donc de l'importance cruciale de l'éducation dans le contexte actuel du bouleversement climatique et apportent différents éclairages à ce sujet. S'appuyant sur l'analyse de corpus de publications de recherche, sur leurs propres travaux, sur leur expérience de recherche empirique, ou plus spécifiquement, sur leur observation par immersion dans divers milieux d'action éducative, ces chercheur.e.s et praticien.ne.s proposent un ensemble de fondements et de pratiques visant à favoriser la pertinence et l'efficacité d'une éducation relative aux changements climatiques.

On observera certains thèmes récurrents à travers leurs contributions. Les auteur.es insistent sur les limites de l'«alphabétisation climatique» par la transmission de connaissances. Et s'ils reconnaissent l'importance d'encourager l'adoption de pratiques écologiques individuelles, ils insistent sur la nécessité d'aller au-delà de ces écogestes et de stimuler plus fondamentalement le développement d'un pouvoir-agir. Celui-ci apparaît indissociable de l'acquisition de "compétences écocitoyennes" associées en particulier à l'exercice de la pensée critique et à l'expérience de l'action réflexive. Les auteur.e.s soulignent également l'importance de la dimension collective de l'action permettant de "briser l'isolement", de mettre en commun les ressources de chacun.e et de stimuler l'espoir dans un contexte où l'écoanxiété devient trop souvent une toile 
de fond existentielle. La charge psycho-sociale et politique de l'action climatique est ainsi évoquée dans plusieurs textes. Enfin, de façon générale, beaucoup d'auteur.e.s déplorent que, malgré son importance cruciale, les systèmes d'éducation n'accordent que peu d'importance à l'éducation relative aux changements climatiques, qui devient pourtant une mission sociétale prioritaire des milieux de formation.

21 Les premiers textes de ce numéro thématique traitent plus spécifiquement de l'ERCC auprès des jeunes: ceux-ci sont en effet interpelés de façon bien particulière par la situation climatique.

Yves Laberge propose d'abord des balises pour définir l'éducation relative aux changements climatiques. Il s'inspire à cet effet « des travaux en pédagogie critique qui mettent en évidence les relations entre pouvoir (étatique ou économique), circulation des savoirs, idéologies et éducation ». L'auteur invite à porter une attention particulière à la façon dont les jeunes apprennent «dans un contexte de libre circulation de l'information ", en particulier via Internet, "un système séduisant et pratique » mais dont l'usage fait particulièrement appel au développement d'un esprit critique. À titre d'exemple, Yves Laberge aborde la relance de la filière nucléaire, que ses promoteurs présentent comme une solution incontournable à la crise climatique actuelle. Ici, l'enseignant.e a un rôle essentiel à jouer pour inviter les jeunes à valider les données recueillies, à faire preuve de discernement, à douter, à contester, à s'interroger en profondeur sur la signification des informations et les intentions qui y sont associées.

Émilie Morin, Geneviève Therriault et Barbara Bader insistent sur l'importance de s'attarder aux dimensions affectives de l'engagement et, plus particulièrement, à ce qu'elles nomment le sentiment de "pouvoir agir " chez les élèves du secondaire. À l'appui de recherches, elles observent que pour contribuer à développer un tel sentiment, l'école devrait aider l'élève «à se sentir bien, important, libre et égal aux autres. Elle devrait faire en sorte qu'il soit entendu et soutenu et devrait contribuer à le rendre fier. Les projets qu'il mène devraient l'aider à sentir qu'il est utile et qu'il change quelque chose pour sa communauté ». Les auteures soulignent aussi l'apport important de la dimension collective des projets et de l'expérience de la réussite.

Pour leur part, Alejandro Pérez Diez, Antonio García Vinuesa, Kylyan M. Bisquert i Pérez et Pablo Á. Meira Cartea s'intéressent à la place de l'ERCC dans la recherche, les politiques climatiques et éducatives, les programmes scolaires, ainsi que dans les manuels scolaires et les pratiques pédagogiques. Observant des lacunes à cet effet, ils soulignent l'importance de développer et de mettre en œuvre un agenda international de recherche éducative sur l'ERCC, ainsi que la nécessité de développer des curricula nationaux qui placeraient la lutte contre le $\mathrm{CC}$ au centre de l'éducation formelle. Pour favoriser le développement des compétences écocitoyennes chez les élèves, ils insistent toutefois sur l'importance « de dépasser la vision limitée promue par les Nations unies, et axée sur la compréhension, la conscience et le comportement individuel»; ils suggèrent plutôt la mise en place de stratégies communautaires.

Toujours en contexte d'éducation formelle au secondaire, Anne Deslauriers se penche sur l'arrimage entre l'éducation artistique et l'éducation relative à l'environnement. Elle propose à cet effet un modèle éducationnel axé sur « la châne interdisciplinaritéréflexivité-criticité en classe d'art», qui ouvre "un espace de questionnement et d'analyse du monde » tant pour les éducateurs que pour leurs élèves. Ce modèle rejoint des éléments clés et pourtant trop souvent négligés du Programme de formation de l'école 
québécoise : ses missions (dont le développement d'un pouvoir agir) et l'ouverture à la transdisciplinarité (via les «domaines généraux de formation » et les compétences transversales). Ici, l'éducation artistique et l'ERE s'intègrent de "manière organique " sans jamais dénaturer l'essence de la discipline des arts plastiques au sein de la formation globale de l'élève et intégrant la prise en compte de questions socioécologiques, dont celle des changements climatiques, « qui redessinent à grande vitesse le présent et l'avenir de l'humanité ».

Valériane Champagne Saint-Arnaud et Ghislain Samson nous transportent dans un contexte d'éducation non formelle, celui du Sommet jeunesse sur le changement climatique organisé dans le cadre des activités de la Fondation Monique-Fitzback au Québec. Ces chercheur.e.s se penchent sur l'évaluation d'une telle activité d'éducation relative aux changements climatiques destinée aux adolescents. Constatant le manque de repères à cet effet, ils proposent un cadre d'évaluation novateur reposant sur douze indicateurs d'efficacité, regroupés autour de trois dimensions du comportement écoresponsable : la motivation, le sentiment d'efficacité personnelle et le passage à l'action, soit l'engagement dans la lutte climatique - au-delà des écogestes. Au terme de la mise à l'essai de ce cadre d'évaluation critériée, les auteur.e.s observent qu'une initiative comme celle du Sommet est de nature à stimuler efficacement le processus d'adoption de comportements écoresponsables. Entre autres, elle permet de contrer l'isolement et d'offrir un certain " apaisement de l'écoanxiété » chez les jeunes. Une fenêtre s'ouvre ici vers le thème de l'article suivant.

Devant le constat que la conscience grandissante de la menace climatique affecte le bien-être psychologique individuel et collectif, Anne Sophie Gousse-Lessard et Félix Lebrun-Paré abordent spécifiquement le phénomène " d'écoanxiété » qu'ils tentent de cerner en croisant les apports complémentaires de la psychologie, de la sociologie et de l'éducation, dans la perspective de mettre au jour des stratégies de régulation de l'écoanxiété et leur prise en compte en éducation: "Malgré la souffrance qu'elle peut causer (...), il ne s'agirait pas de chercher à se débarrasser du sentiment d'écoanxiété, mais plutôt de développer notre capacité à l'accueillir, à composer avec elle, à apprendre de nos craintes et à les mobiliser au travers d'actions signifiantes et significatives ». Les auteur.e.s signalent toutefois des enjeux liés à l'action individuelle et collective. Entre autres, il importe de ne pas faire « l'impasse des rapports de pouvoir qui traversent le corps social». Par une éducation "critique, écologique et émancipatrice ", qui évite de verser dans l'idéologie et le dogmatisme, il s'agit de renouveler le «sens de l'action » en vue de la transformation écosociale.

Examinant les apports et les limites des sciences cognitives au regard de l'éducation relative à l'environnement, Clément Mangin et Anne-Sophie Gousse-Lessard se penchent également sur certains écueils de l'éducation relative à l'action climatique. Entre autres, ils observent que le fait «d'imputer à l'individu la responsabilité de l'inaction» laisse dans l'ombre "l'influence des structures économiques, sociales et politiques ». Ils mettent aussi en évidence que l'adoption d'" une vision pathologique des croyances et attitudes hostiles à l'action climatique » - comme en témoignent les initiatives «thérapeutique» du marketing social - entretient un rapport social «hiérarchique et conflictuel». Ils déplorent que les "politiques publiques comportementales, échappant au contrôle citoyen et se mettant en œuvre par-devers lui, remettent en question les idéaux d'autonomie individuelle et de participation politique». Les auteur.e.s rappellent donc les rôles cruciaux de l'ERE pour le 
"développement de compétences métacognitives ", « la lutte contre l'individualisme " et la promotion d'approches délibératives et démocratiques, en lien avec l'essor d'une conscience éthique et politique.

La section Regards de ce numéro thématique réunit des textes plus courts qui traitent également de divers enjeux de l'éducation relative au changement climatique auprès de différents publics. On y trouve entre autres des propositions de fondements, d'approches et de stratégies transférables à divers contextes.

Constatant d'abord l'importance primordiale et l'urgence de promouvoir l'institutionnalisation de l'ERCC dans les milieux d'enseignement formels, Isabelle Lefebvre et Liliane Dionne déploient un argument de taille pour exiger que les systèmes d'éducation, à tous les ordres d'enseignement, intègrent l'éducation relative à l'environnement et plus spécifiquement, l'ERCC aux programmes d'études. Elles rappellent en effet les différentes ententes internationales (sous forme de Déclaration, Accord, Pacte, Convention et autres) auxquelles les états sont liés et qu'ils se sont engagés à mettre en œuvre. Ces textes relevant du droit international explicitent l'obligation légale des gouvernements de respecter le droit à une éducation de qualité pour tous et le droit à un environnement sain, ce qui implique désormais l'intégration d'une éducation relative au changement climatique dans les programmes de formation.

31 Mélanie Champoux et Adolfo Agundez-Rodriguez portent attention aux dimensions épistémique et éthique de l'ERCC, étroitement reliées entre elles. Ils s'appuient sur les travaux du sociologue portugais Boaventura de Sousa Santos pour mettre en évidence la nécessité «d'élargir notre pensée et notre être-au-monde afin de faire face à l'immense défi civilisationnel que représentent les changements climatiques». L'ouverture vers les "épistémologies du Sud " contribue à une " écologie des savoirs " et favorise "la décolonisation de notre pensée et de nos rapports à l'Autre ». Dans la recherche de nouvelles formes de rapport au monde, valorisant entre autres les apports des cultures occultées, l'ERCC doit se préoccuper de "justice cognitive", étroitement liée à la justice environnementale et à la justice sociale.

Pour affronter la crise climatique, Audrey Groleau, Isabelle Arseneau et Chantal Pouliot proposent d'adopter une approche éducative misant sur les capacités citoyennes. Elles inscrivent leur propos dans le champ de la recherche en didactique des sciences et celui de l'enseignement des technosciences. Plutôt que de tenter de combler ce qui serait un "déficit citoyen" (manque de connaissances, de compréhension et d'intérêt), elles mettent en lumière l'importance de contribuer à la reconnaissance et au développement des capacités permettant aux citoyens.nes « de comprendre les tenants et les aboutissants des questions environnementales et sanitaires contemporaines, de participer aux discussions sociopolitiques et d'agir sur les situations qui les concernent ». En stimulant de telles capacités, « il devient possible de générer l'action sociopolitique, que ce soit en classe (...) ou dans le contexte d'initiatives individuelles ou collectives réalisées dans la communauté ». Les auteures suggèrent des stratégies pédagogiques favorisant un enseignement plus critique et politisé, de nature à soutenir une éducation au changement climatique.

33 Marie-Hélène Desmarais, Rhéa Rocque et Laura Sims s'intéressent également au milieu de l'éducation scolaire. Elles proposent 11 stratégies "pour canaliser l'éco-anxiété » qu'éprouvent les jeunes constatant que leurs actions individuelles, bien que pertinentes et nécessaires, n'ont pas l'effet espéré. Ces stratégies d'adaptation sont classifiées en fonction de trois axes prioritaires : les émotions, le problème et la signification. «Les 
stratégies d'adaptation axées sur les émotions permettent à la personne éco-anxieuse de nommer et de comprendre ses émotions, et de reconnaître sa légitimité ». Les stratégies d'adaptation axées sur les problèmes visent entre autres à augmenter le sentiment d'auto-efficacité et encouragent les élèves à agir. "Les stratégies d'adaptation axées sur la signification visent la recherche de sens en lien avec la crise climatique et ses défis inter-reliés ». Les auteures soulignent l'importance de recadrer la crise et d'y trouver un sens positif. "Il faut offrir aux élèves des occasions pour réimaginer ou réinventer leur futur désiré ». Il faut enfin " fournir des exemples positifs aux élèves de manière à les inspirer ».

Stéphanie Gladu et Manon Curadeau nous amènent en contexte d'éducation non formelle, au Biodôme de Montréal où elles ont conçu et expérimenté une approche éducative visant l'engagement environnemental des citoyennes et citoyens. Les auteures font état de la démarche qui accompagne l'élaboration et l'implantation de cette approche axée sur une "pyramide d'interventions». Il s'agit d'utiliser une information générale - à partir d'une question posée par un.e visiteur.euse - comme prétexte pour parler d'enjeux environnementaux. L'éducateur.rice scientifique "tentera de replacer cette information générale dans un contexte écosystémique. Il.elle cherchera ensuite à faire le lien entre la problématique environnementale qui afflige cet écosystème particulier pour enfin proposer aux visiteur.euse.s des actions qu'ils.elles peuvent entreprendre pour aider à résoudre cette problématique». Les auteures soulignent qu'une telle démarche permet d'accueillir le.la visiteur.euse « là où ileelle est rendu'e " dans son processus personnel d'engagement environnemental. Toutefois, l'implantation de cette nouvelle approche "vers l'action" n'est pas sans défis, dont celui de la formation des animatrices et animateurs en ce sens.

Carole Dupuis présente enfin une initiative majeure de la société civile, le projet Québec ZéN (Zéro énergie Nette) du Front commun pour la transition énergétique, ce vaste ralliement d'organismes et d'acteurs environnementaux mobilisés pour coconstruire le Québec carboneutre de demain, dans un esprit de justice sociale. La question au départ du développement collaboratif d'une "feuille de route» en ce sens était celle-ci : "Connaissant les sources des émissions de GES d'origine anthropique, sachant (au moins en grande partie) comment les éviter, consciente des risques extrêmes qu'elles représentent pour la qualité de vie des générations montantes et même la vie sur terre, pourquoi notre société n'arrive-t-elle pas à poser les gestes qui s'impose ?» La réflexion collective a mené à identifier quatre pistes d'action pour le changement: une vision claire et partagée d'une une société « zéro émission nette "; une information cohérente et exhaustive sur les chemins de la transition; une responsabilisation de tous les acteurs; un mouvement social créé à partir de la base en engageant les acteurs du changement dans la co-construction de celui-ci. Le projet Québec Zen correspond à un vaste projet d'apprentissage collectif pour une action concertée.

L'ensemble de ces textes présente donc un riche gisement de réflexions, d'expériences et de pistes d'action pour développer une éducation relative au changement climatique appropriée à différents contextes. Il est stimulant d'observer par ailleurs le grand potentiel de transférabilité de chacune des différentes propositions (principes, démarches, stratégies) à une diversité de situations éducatives tant en milieu formel que non formel ou informel.

Bonne lecture! 


\section{BIBLIOGRAPHIE}

Agundez-Rodriguez, A. (en rédaction). Modèle transdisciplinaire d'éducation relative aux changements climatiques. Éducation relative à l'environnement, 17(1).

Anderson, K. et Brows, A. (2011). Beyond dangerous climate change : emission scenarios for a new world. Philosophical Transactions of the Royal Society, 369. Consulté sur https:// royalsocietypublishing.org/doi/pdf/10.1098/rsta.2010.0290

Castillo, J-M. (2016). Los negocios del cambio climático. Virus.

Convention-cadre des Nations unies sur les changements climatiques (2015). Accord de Paris. Organisation des Nations Unies.

Convention-cadre des Nations unies sur les changements climatiques (2021). Accord de Glasgow. Organisation des Nations Unies.

Didham, R.J., Declan, D., Klein, J., Thoresen, V.W. (2015). Responsible Living : Concepts, Education and Future Perspectives. Springer International Publishing Switzerland.

Groupe d'Experts Intergouvernemental sur l'Évolution du Climat (2021). Sixième rapport d'évaluation. Programme des Nations Unies pour l'Environnement.

Incropera, F.P. Climate Change : A wicked Problem. Cambridge : Cambridge University Press.

Léveillé, J-T. (2019, septembre 28). Grève mondiale pour le climat : foule record à Montréal. La Presse. Consulté sur https://www.lapresse.ca/actualites/environnement/2019-09-28/grevemondiale-pour-le-climat-foule-record-a-montreal

Meira Cartea, P.A. (2008). Comunicar el cambio climático. Escenario social y líneas de actuación. Ministerio de Medio Ambiente y Medio Rural y Marino.

Pruneau, D., Khattabi, A. et Demers, M. (2008). Éduquer et communiquer en matière de changements climatiques : défis et possibilités. Vertigo, 8 (2), p. 1-9. Consulté sur https:// vertigo.revues.org/4995

Riechmann, J. (2015). Autoconstrucción. La transformación cultural que necesitamos. Madrid : La Catarata.

Sauvé, L. (2014). Au cœur des questions socio-écologiques : des savoirs à construire, des compétences à développer. Éducation relative à l'environnement, 11. Consulté sur https:// journals.openedition.org/ere/662

Welzer, H. (2012). Guerras climáticas. Buenos Aires : Katz Editores.

\section{NOTES}

1. Nous employons ici le terme changement climatique au singulier pour mettre l'accent sur l'aspect global de ce phénomène et de ses conséquences, qui affectent l'ensemble de la planète. Toutefois, au fil du texte et selon le contexte, nous utilisons également l'expression changements climatiques au pluriel pour faire référence aux manifestations et aux conséquences de tels changements selon les régions et pays du monde.

2. https://www.coalition-education-environnement-ecocitoyennete.org/ 
3. https://centrere.uqam.ca/vers-une-strategie-quebecoise-deducation-en-matieredenvironnement-et-decocitoyennete/

4. https://centrere.uqam.ca/

5. https://centrere.uqam.ca/quoi-de-neuf/leducation-relative-au-changement-climatiqueprincipes-approches-et-pratiques-en-milieux-formel-et-non-formel-colloqueinternational-10-11-octobre-2019/

\section{AUTEURS}

\section{ADOLFO AGUNDEZ-RODRIGUEZ}

Université de Sherbrooke

\section{LUCIE SAUVÉ}

Université du Québec à Montréal 